\title{
Wharton's Jelly Mesenchymal Stem Cells as Off-The-Shelf Cellular Therapeutics: A Closer Look into their Regenerative and Immunomodulatory Properties
}

\author{
S. Jyothi Prasanna* and V. Sowmya Jahnavi
}

Manipal Institute of Regenerative Medicine, Manipal University, Bangalore, 560071, India

\begin{abstract}
Mesenchymal stem/stromal cells (MSCs) are isolated from most post-natal tissues and are broadly reported to have similar immuno-phenotype, mesenchymal lineage-differentiation potential and bio-distribution in peri-vascular niches. Thus, several stromal substitutes of bone marrow mesenchymal stem cells (BMMSCs) are being considered for regenerative therapy. Wharton's jelly mesenchymal stem cells (WJMSCs) seem to be the most promising alternative because of easy donor accessibility, high proliferative capacity and greater sample to sample identity.

Recent in vitro and in vivo evidences also support the usage of WJMSCs in tissue repair and regeneration. Key to these observations is secretion of trophic and immune regulatory factors, which aid repair, and resolution of injury. In order to extrapolate these results for clinical usage key questions that need to be addressed are: extrapolation of "allogeneic" transplantation ability of BMMSCs to WJMSCs, survival of allogeneic/xenogeneic WJMSCs in transplantation scenario and actual mechanisms of immune-modulation in an "inflammatory" setting. This review focuses on comparing the in vitro and in vivo studies on immune regulatory properties of WJMSCs and BMMSCs and speculates the usage of WJMSCs for immuno-modulation in a disease scenario. Despite commonalities, different tissue-derived MSCs are reported to have unique gene expression signatures. We would evaluate whether WJMSCs have unique inherent properties, owing to their bio-distribution and primitiveness, as compared to BMMSCs. Further, we debate whether these differences remain conserved on in vitro propagation and impact the immune properties of WJMSCs and speculate the pros and cons of using WJMSCs for allogeneic transplantation.
\end{abstract}

Keyword: Mesenchymal stem cells, immunomodulation, allogenic transplantation.

\section{INTRODUCTION}

The isolation of multi-potent stromal cells from the bone marrow, by Friedenstein in the 1970, led to keen interest in the field of mesenchymal stem cell biology. This was the first report to show that the mesenchyme of a tissue could harbor mesenchymal progenitor/stem cell properties whose potency can be validated in vitro and in vivo [1]. The therapeutic benefits of BMMSCs is now established in several injury and disease models and preliminary data from clinical trials further confirm these claims [2]. In the recent past, several research groups have also identified mesenchymal stem-like populations from different post-natal and pre-natal tissues exhibiting tri-lineage differentiation potential (into osteogenic, adipogenic and chondrogenic lineages) $[3,4]$. These studies promise an easily accessible mesenchymal stem cell alternative to bone marrow. In this context, stem cells isolated from extra-embryonic tissues are of particular interest since they are discarded after birth and would have fewer ethical constraints as compared to BMMSCs.

MSCs are hypo-immunogenic and have been shown to promote regeneration and functional recovery in disease and injury. Key mechanisms in recovery involve immune-

*Address correspondence to this author at the Manipal Institute of Regenerative Medicine, Manipal University, Bangalore, 560071, India; Tel: +91-080-25356661, Ext: 208; Fax: +91-080-25356662;

E-mail: jyothi.prasanna@manipal.edu modulation and trophic effects. The immune privilege properties of adult MSCs make them good candidates for cell based transplantations in an in vivo allogeneic setting $[2,5]$. However, in vitro mixed lymphocyte reactions (MLRs) with fetal mesenchymal stem cells showed immunerecognition unlike BMMSCs [6]. Umbilical cord matrix cells (UCMSCs) are a good peri-natal source of MSCs and their broad immune properties are comparable to BMMSCs [7]. It remains to be analyzed whether they resemble fetal MSCs or adult MSCs with respect to immune-privilege, engraftment and survival under an allogeneic setting. This is of specific relevance because the fetus utilizes unique immune-modulation mechanisms to evade maternal immune surveillance during gestation. This review summarizes the recent developments in Wharton's jelly/Umbilical cord mesenchymal stem cell biology (UCMSCs and WJMSCs are used interchangeably in the manuscript) with specific emphasis on immune properties of WJMSCs in a transplantation environment where inflammation exists. We would debate whether WJMSCs could be considered a replacement to BMMSCs as off-the-shelf therapeutics for transplantation taking into consideration its self-renewal, regenerative and immunomodulatory potential on the whole.

\section{BASIC PROPERTIES OF WJMSCS}

To date, BMMSCs have been considered the gold standard in MSC biology. However, the limitations include an invasive isolation procedure and a higher risk of bacterial 
and viral contamination. In addition, donor age variations amongst samples attribute to differences in the initial yield of isolation and the basic proliferative and differentiation capabilities [8-11]. These factors can affect reproducibility between different bone marrow isolates.

The Umbilical Cord derivatives offer a good clinical alternative to adult MSCs as they are discarded after birth and thus have fewer ethical constraints. Fetal tissues, being less exposed than other adult tissues, have minimal chances of contamination with viral and bacterial antigens. In addition, donor age variations are minimal with cord derived stromal cells.

MSCs have been isolated from different regions of the cord; cord blood, umbilical cord matrix and the peri-vascular region. Out of these cord derivatives, Umbilical cord matrix or the WJMSCs, which include the inter-vascular and subamniotic matrix cells, offer better clinical utility. The isolation frequency of colony forming unit-fibroblasts (CFUFs) from each cord matrix is extremely high and a delay in processing until 48 hours does not impact isolation, in contrast to cord blood, where a small delay of 5 hours can reduce the isolation efficiency to $60 \%$. Umbilical cord blood derived MSCs are rare and recovery is also highly variable because of inconsistency in initiating cultures and the required expansion time to reach clinical numbers. Approximately $1 \times 10^{4}$ cells can be initially isolated from just $1 \mathrm{~cm}$ of the cord sample. However, only 1-10 MSCs have been isolated from $1 \times 10^{6}$ mononuclear cells (MNCs) from bone marrow and $10^{3}$ cells per total sample of cord blood [12-14 and reviewed in 15 and 16].

Human umbilical cord perivascular cells (HUCPVCs) isolated after dissecting out the umbilical vein/arteries, despite having a high proliferative potential, have limited transdifferentiation potential $[14,16,17]$. Also, marker expression, along with differentiation assays indicate that the mesenchyme becomes more differentiated and mature as we move from the sub-amnion region to the perivascular region [14]. Thus, WJMSCs could be a better MSC source than HUPVCs amongst the cord stem cell derivatives.

Both, enzymatic and non-enzymatic methods, have been used to isolate WJMSCs. Irrespective of minor differences in isolation procedures, WJMSCs have been consistently reported to have high ex vivo proliferation index and low population doubling times, which makes them more expandable before reaching senescence [14, 18-20]. Approximately, 300 fold expansions are reached within 6-7 passages without any sign of abnormal karyotype. Moreover, different isolates are more age-matched [14]. Clinical usage of MSCs requires ample amount of cells at a lower passage to negate out karyotypic instabilities and epigenetic damages, which are predicted, due to replicative aging. WJMSCs show no signs of transformation like loss of anchorage dependence, contact inhibition and serum dependence over several passages [12, 14]. All these attributes make WJMSCs a reproducible source for banking and clinical grade expansion.

\section{STEMNESS AND SELF-RENEWAL OF WJMSCS}

WJMSCs are more primitive than their bone marrow counterparts probably due to less temporal separation from fetal tissues at birth and thus might contain resident stem cell populations with enhanced potency. Wharton's jelly is derived from extra-embryonic mesoderm and partly from the embryonic mesoderm [21, 22]. Porcine WJMSCs have been shown to express low levels of pluripotency markers like Oct4A (the Oct4 isoform involved in self-renewal), Nanog, and Sox2 and depict alkaline phophatase positivity [23]. Unlike BMMSCs, they express functional human telomerase reverse transcriptase (hTERT) activity like embryonic stem cells (ESCs), yet the cells are not transformed [14, 24, 25]. In addition, they have a high percentage of Hoesct-excluding cells and show an aldehyde dehydrogenase-positive phenotype as displayed by primitive hematopoietic stem cells (HSCs) [12, 23].

However, despite these primitive characteristics, no teratoma formation has been reported on transplantation of WJMSCs in several animal models. WJMSCs, interestingly, express a high percentage of tumor suppressor genes as compared to other classes of MSCs [26]. Comparison of transcriptomes of WJMSCs and BMMSCs indicated a substantially higher fold expression of ES cell related pluripotency markers like Nanog, DNMT3B, GARB3, Lin28 and other stemness markers like ABCG2, CD9, KIT etc in WJMSCs [19, 26, 27]. Despite basal level expression of pluripotency markers, high expression of tumor suppressor genes might counterbalance and confer WJMSCs with low propensity to turn neoplastic. In addition, few early lineage markers specifically related to endoderm are upregulated in WJMSCs, both in early and late passages, as compared to BMMSCs [19, 28]. A higher expression of early ectoderm markers were also reported in WJMSCs in this study. It remains to be validated whether these differences in the transcriptome reflect in differential trans-differentiation propensities to endodermal and ectodermal lineages. In general, transcriptome analysis within ESCs, embryonic carcinoma cells (ECCs) and adult MSCs consistently report a higher number and higher expression of stem cell and germ lineage markers [12, 25, 26, 29]. Also, stem cell properties and gene expression signatures are retained in WJMSCs till late passages, as compared to BMMSCs where stark differences in stemness signatures had been reported after 3 5 passages [30].

WJMSCs are a primitive source of stem cells with stem cell characteristics in between an ESC and a more mature adult stem cell in the developmental hierarchy. However, the stemness of WJMSCs has to be yet proven using clonal in vivo self-renewal and multipotency assays in a similar manner like HSCs to make definitive statements.

\section{STROMAL SUPPORT PROPERTIES OF WJMSCS}

WJMSCs provide a supportive stromal niche/microenvironment for several primitive stem cell populations. WJMSCs and conditioned medium from these cells have been used to support human ES and equine ES-like cell colonies [16]. In addition, UCMSCs also provide stromal support to HSCs and spermatogonial stem cells [31, 32]. This could be explained because of a probable migration of these cells along with primordial germ cells and fetal HSCs to the aorta-gonad-mesonephros (AGM) region during development [33]. WJMSCs, like BMMSCs, constitutively 
secrete hematopoietic cytokines. Co-culture with WJMSCs enhanced the in vivo homing capacity of $\mathrm{CD} 34^{+}$umbilical cord blood (UCB) cells through induction of homing adhesion molecules [31]. Comparative studies on HSC-support suggest that BMMSCs have higher hematopoietic supportive ability but WJMSCs support long-term hematopoiesis [31]. WJMSCs have also been shown to support islet cell survival and function in co-culture models before transplantable Islet cell clusters (ICC) levels are reached [34]. Further studies for understanding the physiological role of MSCs in various niches at different developmental stages are required to predict whether they can specifically support different stem cells.

\section{TRI-LINEAGE DIFFERENTIATION POTENTIAL AND TRANS-DIFFERENTIATION TO ECTO- AND ENDODERM-DERIVED LINEAGES}

WJMSCs exhibit the tri-lineage differentiation potential, which authenticates them as functional MSCs according to International Society for Cellular Therapy (ISCT) guidelines [35]. WJMSCs, like BMMSCs, share similar osteogenic differentiation pathways with respect to key osteogenic gene signatures. However, both in vivo and in vitro results indicate less mineralization $[36,37]$. In a sub-cutaneous implantation model, ectopic bone formation was confirmed by von Kossa staining but osteogenesis and mineralization was weaker as compared to BMMSCs. However, bone fracture healing in vivo was equally potent with both WJMSCs and BMMSCs [37]. These contradictions could be because of differential efficacy of differentiation cocktails and compatibility of scaffolds with these two cell types.

Comparison of chondrogenic differentiation with BMMSCs and WJMSCs indicated higher production of collagen from the latter when differentiation was performed with microsphere based scaffolds or polyglycolic acid (PGA) scaffolds. Although the expression and synthesis of Collagen II and expression of pro-chondrogenic markers like Sox9, Runx2 was lower than BMMSCs in in vitro assays WJMSCs seem to be more compatible with tissue engineering principles in vivo for osteochondral regeneration [38].

WJMSCs have been reported to have similar basic adipogenic gene signatures when exposed to similar inductive stimuli; but they required a longer time in inductive media to show robust oil droplet formation upon oil O red staining [39]. We, along with other groups, have reported that WJMSCs derived adipocytes have smaller oil droplets as compared to the evenly sized large oil storing vacuoles detected with BMMSC-derived adipocytes. However, the percentage of cells staining for oil $\mathrm{O}$ red was similar in both the cell types $[14,20]$. It remains to be checked whether adipocytes derived from WJMSCs are terminally differentiated or are stuck at the pre-adipocyte stage.

Neural differentiation has been shown from UCMSCs but the efficiency of generation of neural cells varied in differentiation protocols using different chemical and developmental cues. Under identical in vitro induction conditions, an earlier generation of neurospheres was noted from UCMSCs as compared to BMMSCs [40, 41]. UCMSCs produce higher quantities of neurotrophic factors and growth factors for endothelial cells than BMMSCs, both constitutively and upon neuronal differentiation [12, 14, 18, 42-44]. In cerebral ischemia models, UCMSCs not only migrated to site of injury but also differentiation into glial, neuronal and a vascular endothelial cells was noted [45]. It is speculated that the recovery in brain injury models could be attributed to neuro-protective effects of UCMSCs and/or increased plasticity of endogenous stem cells along with enhanced vasculogenesis at the site of injury upon UCMSC transplantation. UCMSC transplantation could cause behavioral recovery in a hemi-Parkinson rat model by rescuing degenerating dopaminergic neurons and trophic factors were implicated in rescue $[12,46]$. In fact, UCMSCs have been reported to be more potent in repairing photoreceptor damage and anatomically rescuing retinal damage than placental and adult bone marrow-derived MSCs [18]. However, transplantation of in vitro differentiated Tyrosine hydroxylase positive $\left(\mathrm{TH}^{+}\right)$cells by $\mathrm{Fu}$ et al., in a mouse Parkinson disease model caused behavioral recovery, also these cells did persist for 4 months in vivo [47].

WJMSCs have also been shown to differentiate into endoderm lineages [48-50]. Anzalone et al. have reviewed the differentiation of various mesenchymal stem cells (including WJMSCs, BMMSCs, Umbilical cord blood MSCs and adipose tissue derived MSCs) into pancreatic lineage in detail [51]. A comparative study of BMMSC and WJMSCs to pancreatic islet cell lineage, under identical induction conditions, reported the formation of larger beta-islet cell clusters and concomitantly a higher insulin production was detected in the WJMSC trans-differentiated cultures [50].

Not only WJMSCs but even WJMSC-derived differentiated islet clusters and hepatocyte-like cells caused functional recovery in Streptozotocin (STZ)-induced diabetic rats and allo-transplantation in the liver respectively $[48,52]$. In these studies, there was no rejection of transplanted cells even in the absence of immune-suppressants.

All these evidences point out that the WJMSCs can successfully differentiate into cell types of different lineages and cross lineage barriers on exposure to appropriate developmental / micro-environmental clues. It still needs to be definitively resolved whether, the trophic factors secreted cause majority of recovery, by facilitating survival of endogenous stem cells and degenerating tissue or the transplanted differentiated derivates functionally integrate into the host tissue and have a more proactive role in in vivo disease models. Most in vitro data also suggest the lack of attainment of full functional maturity of differentiated derivates even though a plethora of mature markers are expressed. It remains to be understood whether functional maturity is attained in the in vivo diseased niche.

\section{IMMUNOGENICITY OF WJMSCs}

\section{Expression of Immunologically-Relevant Ligands on BMMSCs and WJMSCs}

Mesenchymal stem cells have been shown to be hypoimmunogenic owing to the lack of HLA-DR and lack of costimulatory ligands implicated in activation of both $\mathrm{T}$ and $\mathrm{B}$ cell responses. MHC-Class I molecules are expressed at low levels and this could be a mechanism to protect them from 
Natural killer cell (NK)-mediated lysis. Treatment with proinflammatory cytokines like Interferon $\gamma(\mathrm{IFN} \gamma)$ results in the up-regulation of both HLA-Class I and HLA-DR but costimulatory molecules are not induced [20,53]. This could result in sending only a partial signal to allo-reactive $\mathrm{T}$ cells resulting in an anergic state. BMMSCs failed to elicit an allogeneic response even after tri-lineage differentiation. MHC class II levels were not induced on the mesenchymal differentiated derivatives even after pretreatment of MSCs with IFN $\gamma$ prior to differentiation [54]. Even though the overall expression of immune-stimulatory ligands on WJMSCs remains similar to that of BMMSCs their induction with pro-inflammatory cytokines might differ. We and others have reported that HLA-DR is induced substantially in BMMSCs with IFN $\gamma$ treatment but in WJMSCs the induction is very negligible $[20,55]$. It remains to be ascertained whether, at higher thresholds and duration of exposure to IFN $\gamma$, substantial induction of HLA-DR is achieved. In addition, no expression of co-stimulatory ligands - CD80, CD86 and B7-DC was observed on WJMSCs even after IFN $\gamma$ treatment $[20,56]$. In fact, like BMMSCs, WJMSCs express constitutively the negative co-stimulatory ligand, $\mathrm{B} 7-\mathrm{H} 1$, and the levels are majorly induced upon IFN $\gamma$ stimulation [56]. There are no in vitro reports on whether differentiated derivatives from WJMSCs retain their immune-privilege.

\section{Evidences on Acceptance of WJMSCs upon Allo- /Xenogeneic Transplantation}

There are conflicting reports on the immunogenicity of BMMSCs in vitro as well as in vivo. BMMSCs seem to be recognized by allogeneic and xenogeneic lymphocytes and trigger substantial amount of IL-4 and IFN $\gamma$ [55]. Mild proliferation of allogeneic lymphocytes was observed in cultures with BMMSCs [57]. In fact, allogeneic murine BMMSCs were rejected by MHC class I and class II mismatched recipient mice [58]. Even in instances where murine MSCs did not elicit an immune response in vivo immune suppression was provided and a lymphocytic infiltrate was observed at the site of transplantation [59]. Liechty et al., had shown that Xenografted human BMMSCs survive and differentiate upon in-utero transplantation in sheep utreus [60]. The survival of human BMMSC in this model could be attributed to the immune privilege status of the site of transplantation and the lack of a mature immune system to recognize the xenograft. Therefore, the universal immune privilege property of BMMSCs is still a debatable issue. ESCs and their derivatives upon transplantation not only generate teratomas, they also trigger robust immune responses under both allogeneic and xenogeneic transplant conditions [61, 62]. In contrast, Wharton jelly MSCs, both at early and late passages, do not elicit a proliferative response from either xenogeneic splenocytes or purified allogeneic responder $\mathrm{T}$ Cells in a one way immunogenicity reaction [7]. We have also observed mild lympho-proliferative responses to BMMSCs but none to WJMSCs upon co-culture with human peripheral blood mononuclear cells (PBMCs) [20]. WJMSCs, as well as their differentiated progenitors, did not elicit any immune response under xeno-transplant settings even in the absence of any immune suppression. Porcine UCMSCs not only survived but also expanded up to 4 weeks and generated $\mathrm{TH}^{+}$neurons which express pig specific neuronal markers in a rodent model of Parkinson's disease [63]. In addition there was no immune infiltrate observed at the site of transplantation. Similarly, human WJMSCS survived for 16 weeks post transplantation in a rat spinal cord injury model in immune competent rats in the absence of any immune suppressive drugs [46]. In a study where the immunogenicity of human BMMSCs and UCMSCs were compared in vivo in immuno-competent mice, BMMCs exhibited a quicker rejection response. However, in immune deficient SCID-beige mice, cell survival was prolonged and similar in both the mesenchymal stem cell types [55]. Interestingly, fetal liver derived MSCs also elicited mild lympho-proliferative responses at high dose ratios in comparison to adult MSCs [6]. All these in vitro and in vivo findings indicate that WJMSCs are less immunogenic than BMMSCs as well as fetal MSCs making them more amenable for allogeneic as well as xenogeneic transplantation.

\section{IMMUNOMODULATORY PROPERTIES OF WJMSCs}

Mesenchymal stem cells from bone marrow have been widely reported to attenuate mitogen driven as well as alloantigen or specific antigen driven $\mathrm{T}$ cell responses in a dose dependent manner in vitro [64]. MSCs have been to shown to equally inhibit $\mathrm{CD}^{+}, \mathrm{CD} 8^{+}, \mathrm{CD}^{+}$and $\mathrm{CD}^{+}$ subsets [65]. In a study, where the mitogen induced $\mathrm{CD}^{+} \mathrm{T}$ cell responses were assessed in the presence of different tissue-derived MSCs, WJMSCs exhibited a prominent suppression even at very low dose range as compared to BMMSCs $[20,66]$. These differences in the immune modulatory properties could be due to the differences in the intrinsic tissue source rather than a simple donor to donor and experimental variation. In addition, WJMSCs suppress allogeneically-stimulated $\mathrm{T}$ cells to a greater extent than either BMMSCs or adipose tissue-derived MSCs (ADMSCs). In all these tissue-derived MSCs, irrespective of $\mathrm{T}$ cell stimuli, an expansion of $\mathrm{CD} 4^{+} \mathrm{CD} 25^{+} \mathrm{Foxp}^{+}$Treg lymphocytes was observed which resulted in an overall attenuation of $\mathrm{T}$ cell effector responses. Treg expansion and maintenance was observed in all $\mathrm{MSC} / \mathrm{T}$-cell co-cultures irrespective of whether they are freshly purified or $\mathrm{CD}^{+}$ recruited Treg cells. Similarly, when ESCs were cotransplanted with MSCs in an immuno-competent rat myocardial infraction model, the immune infiltrate constituted mainly Foxp3 positive Treg cells as compared to ESC alone transplanted rats. However, in this study, the exact role of immune suppressive mechanisms of MSCs in better disease recovery is not clearly dissected [67]. Fetal liver-derived MSCs suppress lympho-proliferative responses to mitogens but do not attenuate allo-proliferative responses [6]. This could be due to lack of immunological maturity till the third trimester. In this context, peri-natal MSCs, like that of WJMSCs, not only seem to attenuate lymphoproliferation more robustly than BMMSCs, but also the regulation is stimuli-independent unlike fetal MSCs. Allogeneic BMMSCs did not exhibit any suppression of PBMCs stimulated with recall antigens [57]. This is beneficial clinically for the host under MSC transplant setting, as MSCs will not meddle with the host immune responses. It remains to be studied whether WJMSCs differ from BMMSCs in their response to cell proliferation to recall antigens like Tetanus Toxoid. 
BMMSCs have been shown to affect the maturation and activation of dendritic cell (DC) precursors [65]. WJMSCs, when cultured with $\mathrm{CD} 14^{+}$monocytes, inhibited their differentiation into mature DCs in a contact-dependent manner. WJMSC-co-cultured monocytes were shown to be locked in an immature DC phenotype and the up-regulation of co-stimulatory ligands was blocked in the co-cultures [56]. Thus, WJMSC might also indirectly affect $\mathrm{T}$ cell allogeneic responses though attenuation of DC functions. Though the immune-regulatory role of MSCs can be put to translational use to control erratic inflammatory responses caution should be exercised regarding the disease history of the patient. This is of particular relevance because BMMSCs have been shown to promote the tumorigenicity of B16 melanoma tumor cells. B16 melanomas are rejected by antitumor mechanisms in an immune-competent host however, co-transplantation with BMMSCs favored tumor growth [59]. The ability of MSCs to support tumorigenesis could be harmful when masked or benign tumor beds are latent in the host where MSC transplantation has to take place.

\section{Mechanisms of Immune-Modulation by WJMSCs}

BMMSCs have been shown to affect the differentiation, maturation and activation of purified immune populations like T cells, B cells, dendritic cells and NK cells $[65,68]$. Studies with purified populations of immune cells and tracing their activation and effector functions closely in presence of WJMSCs are limited. We have tracked the proinflammatory cytokine secretion patterns kinetically in cocultures of WJMSC/BMMSC with PHA-activated lymphocytes. A change in the threshold and kinetics of IL-2 secretion was observed only with BMMSCs and not with WJMSCs. Additionally an early activation of negative costimulatory ligands like CTLA4 on peripheral blood lymphocytes was observed more evidently with WJMSCs co-cultures [20]. These results suggest that although both WJMSCs and BMMSCs cause suppression of mitogen induced lymphoproliferation the populations being suppressed might vary. Moreover, immune-suppression in BMMSCs has been reported to be unrestricted to major histo-compatibility complex (MHC) and occurs by both autologous and allogeneic sources [54]. However, contradictions exit. Addition of donor derived BMMSCs as compared to allogeneic BMMSCs enhanced immune response in a bone marrow allogeneic transplant setting and elicited a memory $\mathrm{T}$ cell response [69]. It is imperative to understand whether immune suppression is a transient state resulting due to anergy of immune cells, that can be rescued, or it causes permanent damage to immune homeostasis in a clinical transplantation setting.

Detailed studies have been performed with BMMSCs to check the role of contact dependent and contact independent mechanisms in mediating immune-suppression. Soluble factors secreted by MSCs have been implicated to have major role as the inhibition is stimuli independent in most cases [70]. However, contact dependent mechanisms cannot be ruled out, as the threshold of suppression is more upon contact with MSCs and occurs at a higher dose ratio. Soluble factors implicated in MSC-mediated immune-modulation include Nitric oxide (NO), Indoleamine 2, 3-dioxygenase (IDO), Heme Oxygenase 1 ( $\operatorname{Hmox} 1)$, secretion of anti- inflammatory cytokines like IL-10, Transforming growth factor- $\beta$ (TGF- $\beta$ ), Hepatocyte growth factor (HGF), IL-6 and Prostaglandin E2 (PGE2) [5, 65, 71-73]. Li et al. have reported that MSCs, through soluble factors, alter the migratory activity of $\mathrm{T}$ cells into secondary lymphoid organs in vivo through induction of a naïve $\mathrm{T}$ CD62 $2^{+} / \mathrm{CCR} 7^{+}$ phenotype both in vitro and in vivo [74]. In addition, MSCs alter the maturation status of DCs and inhibit their localization into secondary lymphoid organs further trapping the naïve $T$ cells in an unactivated state [74]. Since immune regulation in vivo cannot be examined in isolation, more studies tracking the homing of each immune population upon transplantation of WJMSCs need to be performed to check why WJMSCs escape immune surveillance in vivo.

Specialized immune tolerance mechanisms have been implicated at the maternal-fetal interface. These mechanisms depend on expression of HLA-G and expression of immune suppressive cytokines like Leukemia inhibitory factor (LIF) $[75,76]$. WJMSCs, being isolated from a peri-natal source, could exhibit immune evasion mechanisms prevalent at the fetal maternal interface. In fact, WJMSCs express higher constitutive as well as IFN $\gamma$ inducible levels of LIF than BMMSCs and the suppression of lymphoproliferation can be rescued by blocking LIF in co-cultures [77]. Similarly, we have reported higher levels of both constitutive and IFN $\gamma$ inducible HGF in WJMSCs over BMMSCs [20]. Additionally, UCMSCs have been shown to constitutively express high levels of the immune-suppressive HLA-G isoform, HLA-G6 but not HLAG5 [7]. BMMSCs express HLAG5 isoform constitutively but its expression is not induced by IFN $\gamma$. HLA-G5 secretion has been directly implicated in induction of Treg phenotype and suppression of NK cell production of IFN $\gamma$ in BMMSC co-cultures [78]. Fetus expresses high levels of HLA-G to inhibit maternal alloreactivity. However, the exact role of immune-suppressive HLA$\mathrm{G}$ isoforms in influencing immune suppression needs to be evaluated in detail. It's possible that the HLA G-6 isoform has a specific role in WJMSC-mediated immune suppression.

Although the major secretary profiles of different tissue derived MSCs are similar, WJMSCs and cord blood MSCs only secrete IL-12, IL-15 and PDGF-AA. WJMSCs did not secrete VEGF in comparison to other adult MSC sources [79]. VEGF has been implicated in affecting DC maturation status. These cytokines affect the activation status of different immune populations. The precise meaning of these differences however, needs to be understood in WJMSC/ immune cell co-cultures. The putative mechanisms involved in impacting WJMSC immune regulatory roles are illustrated in Fig. (1).

\section{IMMUNE-MODULATION BY MSCS IS CONTEX- TUAL}

Several discrepancies in the literature have been reported regarding the immune modulatory state of MSCs in vivo as compared to in vitro studies. Growing evidences in the field also indicate that immune-suppression by MSCs is not constitutive but is induced in specific cellular and environmental milieus (Fig. 2). Immune modulatory state of MSCs in vivo remains a quasi-stable state which depends on the 


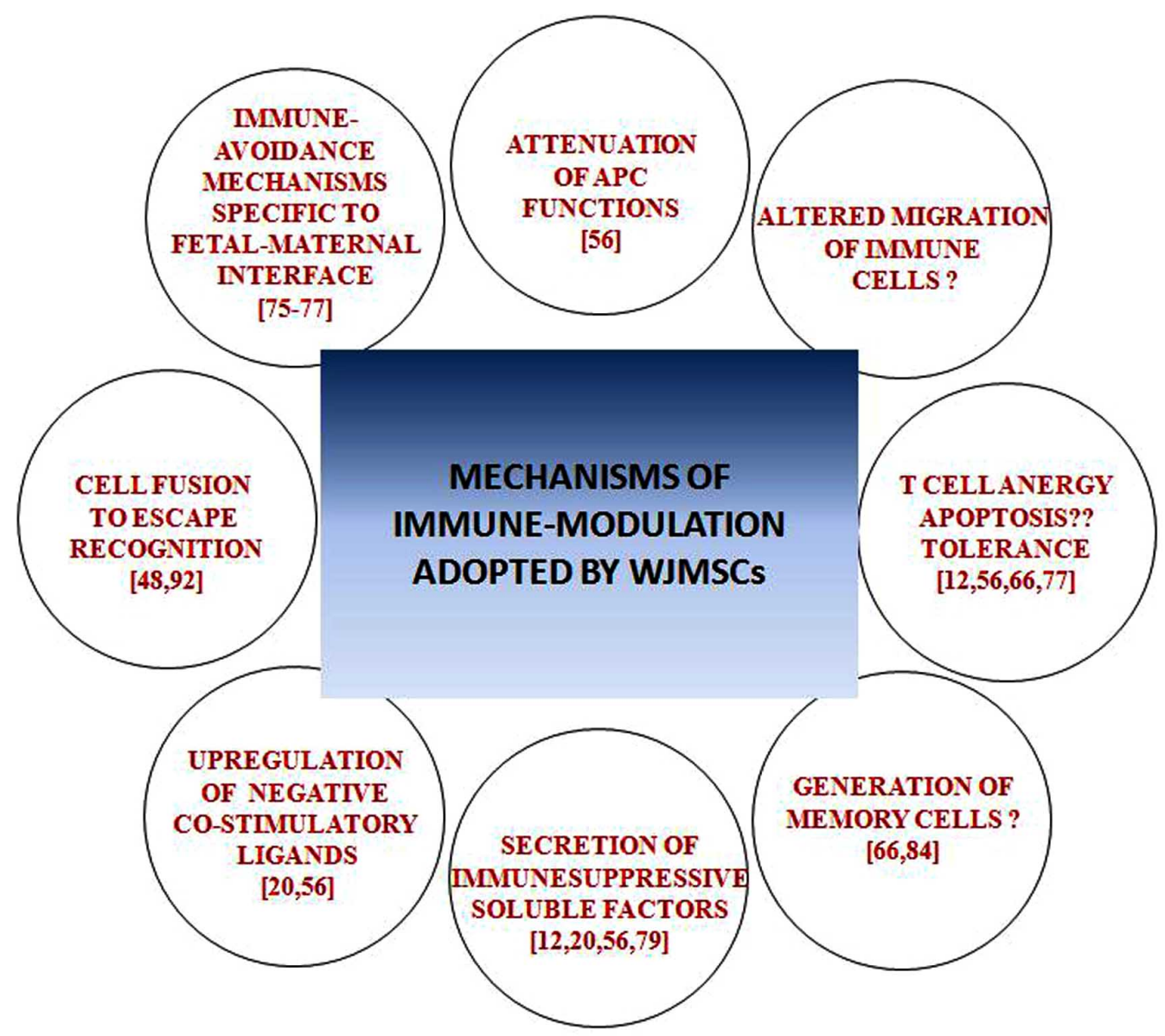

Fig. (1). Mechanisms implicated in WJMSC mediated immune-modulation. Respective references are indicated within brackets.

several factors which include: a) Activation state of $\mathrm{T} / \mathrm{B} / \mathrm{DC} /$ NK cells at time of interaction with MSCs, b) The inflammatory environment persistent at the time of transplantation, c) The actual ratios of the interacting populations, d) the general immune status of the site at which MSCs are being transplanted, e) threshold of stimulus used to trigger the immune populations, $f$ ) the origin of MSC (murine or human or porcine), g) source of MSCs (tissue origin).

\section{Role of Cell Dosage in Affecting Immune-Modulation}

Most studies suggest that immune suppression by MSCs is effective enough to attenuate immune responses over a broad dose range. However, few studies also report that low doses of MSCs can actually enhance lymphocyte proliferation. At low concentration, MSCs seem to support T cell proliferation $[66,80]$. This effect was observed irrespective of whether the $\mathrm{T}$ cells were derived from cord blood (CB) or adult peripheral blood (PB). At lower doses, a switch towards a CD45RO phenotype was observed pointing towards a probable generation of memory $T$ cells. This study also indicates functional differences in interaction of MSCs with both $\mathrm{T}$ cell sources. CB $\mathrm{T}$ cells are phenotypically immature; expressing majorly CD45RA where as PB T cells express equal proportions of CD45RA and CD45RO forms. Populations exhibiting a CD45RA phenotype were less inhibited by MSC [66]. We have also observed differences in proportions of CD45RA and CD45RO populations generated in co-cultures of WJMSCs /BMMSCs and phytohaemagglu- tinin (PHA) stimulated $\mathrm{T}$ cell blasts in direct contact. Cocultures of low dose BMMSCs and IFN $\gamma$. stimulated BMMSCs elicited the generation of a transient CD45RO population. However, such an effect was not observed by both IFN $\gamma$ treated and untreated WJMSCs. Therefore, it is possible that WJMSCs enrich for different $\mathrm{T}$ cell subsets during an activation response as compared to BMMSCs [20]. This could explain the mechanistic differences observed in BMMSC and WJMSC mediated immune suppression as discussed before. In addition, we have also observed mild proliferation in low dose WJMSC: PHA stimulated PBMC co-cultures. A similar effect was also observed with human amnion derived MSCs [81]. So it seems like the immune privilege status of the different tissue derived MSCs as well as the activation status of interacting $\mathrm{T}$ cell populations (naïve versus memory versus pre-activated phenotype) is important in modulating the MSC immune regulatory state. Since MSCs have been shown to have a veto-like power in affecting alloresponses it is possible that lower versus higher dose interactions convey different signal strengths/inputs to the responding $\mathrm{T}$ cells thus shifting the balance towards higher activation or suppression.

\section{Role of Soluble Factors and an Inflammatory Microenvironment in Facilitating WJMSC-Mediated Immune-Suppressive Mechanisms}

Many soluble factors have been implicated in MSC immune suppressive mechanisms; however, none of them 
seem to have a non-exclusive role. Abrogation of the immune suppressive factors in MSC-lymphocyte co-cultures leads to only a partial rescue of suppression. In fact, the activation status of the interacting lymphocyte and the general inflammatory state of the local milieu is more important. This has been definitively proved in IFN $\gamma$ receptor K/O and iNOS-/- murine MSCs where suppression was significantly diminished [73]. The immune suppressive state of MSCs has been shown to occur in a narrow window of IFN $\gamma$ activation [82]. Several reports are in consensus regarding the definitive role of IDO and tryptophan depletion by MSCs in attenuation of T cell proliferation. IDO is not expressed constitutively in both BMMSCs and WJMSCs and is induced only upon exposure to pro-inflammatory cytokines like IFN $\gamma$ and TNF $\alpha[20,56,79]$. In fact, a substantial increase in Treg expansion was seen only in UCMSC-T cell co-cultures in presence of IFN $\gamma$ in comparison to UCMSC-T cells alone. T cell suppression by WJMSCs was rescued by addition of an IDO inhibitor in this study, emphasizing the role of extraneously added IFN $\gamma$ and IFN $\gamma$ generated during an allogeneic response in priming the immunomodulatory mechanisms in WJMSCs [56]. Similarly, although expression of B7H1 was implicated as a potent inhibitor of allo-T cell responses in WJMSC-T cell co-cultures, the role of $\mathrm{B} 7 \mathrm{H} 1$ was exaggerated in IFN $\gamma$ treated WJMSC co-cultures. A higher rescue of T cell proliferation was observed in IFN $\gamma$ co-cultures than untreated co-cultures by $\mathrm{B} 7 \mathrm{H} 1$ blockage despite the fact that $\mathrm{B} 7 \mathrm{H} 1$ was constitutively expressed in WJMSCs [56]. Our studies also suggest an enhanced suppression of MLRs by third party WJMSCs primed with IFN $\gamma$ [20]. These studies are strengthened by observations in graft versus host disease (GVHD) models where IFN $\gamma$ was shown to be absolutely critical for BMMSCs to ameliorate disease [83]. Cho et al., evaluated the immunogenicity of unactivated and IFN $\gamma$ pre-activated porcine WJMSCs by both subcutaneous (highly immunogenic route) and intravenous injections across full MHC barriers. Single injection of unactivated WJMSCs did not trigger adaptive immunity. However, immunogenicity was induced when WJMSCs were pretreated by IFN $\gamma$ or when unactivated MSCs were injected at an inflammatory site. Repeated injections of WJMCS at the same site also resulted in immunogenicity probably due to creation of repeated injury and inflammation at the same site or due to heightened memory responses during repeated injections [84]. Upregulation of HLA-DR could be one of the mechanisms for sensitization. To avoid immunogenicity and sensitization during repeated injections, different strategies like increasing the time between multiple injections, performing repeated transplantation at disparate sites and including concurrent immunosuppressive regimens has been tested in the porcine preclinical model. This study emphasizes on the use of immunosuppressive drugs during each injection to avoid a heightened immune response during repeated injections [85]. These results should also be extrapolated carefully for human WJMSCs from a clinical perspective as species specific differences in immune-modulation mechanisms exist [reviewed in 5]. For e.g., in contrast to murine BMMSCs, human BMMSCs lacking IFN $\gamma$ receptor and IDO still retained immune modulatory properties. In addition, induction of iNOS was minimal in human BMMSClymphocyte co-cultures in contrast to murine BMMSCs.
Similarly, murine and human BMMSCs have been shown to exhibit differential responses to induction of immunesuppressive factors upon treatment with pro-inflammatory cytokines, IFN $\gamma$ and TNF $\alpha[20,86]$.

\section{Activation/Differentiation State of the Interacting Immune Cells and WJMSCs Influences Immune Outcome}

In a graft versus host disease and a transplantation scenario across allogeneic and xenogeneic settings, the initial responders constitute the innate immune components like the NK cells, monocytes and dendritic cell populations which trigger the next phase of adaptive immunity.

The interactions of BMMSCs with NK cells seem to be reciprocal. The activation status of BMMSCs as well as the activation status of interacting $\mathrm{NK}$ cells influences the outcome of immune suppression. MSCs express NK receptors in an IFN $\gamma$ inducible manner. BMMSCs inhibited IL-2 induced proliferation of resting NK cells but surprisingly both autologous and allogeneic MSCs were killed by activated NK cells. However, MSCs pre-activated by IFN $\gamma$ escaped activated NK mediated killing due to induction of higher thresholds of HLA-E and NK inhibitory ligands [87, 88]. Fetal liver-derived MSCs (fMSCs) were rejected by IL-2 activated NK cells and the rejection response was stronger for fMSCs than adult MSCs in both allogeneic and autologous contexts. However, IFN $\gamma$ stimulation of fMSCs rescued them from NK cell mediated killing similar to adult MSCs. Importantly, the expression of NK ligands differed in adult MSCs and fMSCs and both were killed by pre-activated NK cells through distinct pathways. Fetal MSCs were killed by a TNF-related apoptosis-inducing ligand (TRAIL) pathway whereas adult MSCs were susceptible to Fas-FasL mediated killing [89]. In a study where both autologous and allogeneic WJMSCs were used as feeders to expand NK cells in the presence and absence of NK growth factors, an enhanced proliferation of NK cell precursors was observed irrespective of the MHC matching. WJMSC feeders were rejected during the first week of culture itself in this study [90]. However, it is not clear whether a difference in kinetics of rejection is seen in growth factor supplemented NK cell cultures over NK cells alone. The role of a pre-activated inflammatory environment for WJMSC to escape NK mediated killing has to be evaluated analogous to BMMSCs. This is of importance because WJMSCs could be killed by NK cell through distinct mechanisms than what is utilized for BMMSCs and fMSCs. Interference with these specific mechanisms could result in a transplant acceptance for a longer time period.

DC/monocyte lineage immune populations also play an important role as an initial defense mechanism against nonself antigens and priming with DCs is important to trigger cytotoxic $\mathrm{T}$ cell adaptive responses. WJMSCs inhibit DC maturation and activation irrespective of whether the contact happens at the $\mathrm{CD} 14^{+}$monocyte level or the $\mathrm{CD} 14^{-}$ immature DC level suggesting that UCMSCs interfere with the functions of key antigen presenting cells (APCs) which might trigger a cytotoxic $\mathrm{T}$ cell response. Cell contact enhanced the efficiency of suppression implicating the role of both soluble immune suppressive factors as well as cell surface ligands for e.g., B7H1 in DC suppression [56]. The 
precise role of DC suppression by WJMSCs in an in vivo xeno / allotransplant setting has to be established.

In STZ immuno-competent mouse model of Diabetes mellitus pre-differentiated islet like clusters were shown to be more effective in ameliorating disease physiology over undifferentiated WJMSCs upon transplantation [48, 91]. In the model where WJMSC derived islet like clusters were injected into the liver, existence of fused cells staining for human nuclei was documented suggesting that immuneescape could be due to a fusion event [48]. In a Non-obese Diabetic (NOD) mouse model, retro-orbital injection of differentiated insulin producing cells from human WJMSCs rescued glucose homeostasis and a similar fusion event in the liver was reported [92]. When WJMSC derived islet like clusters were injected under the kidney capsule in STZ mice a reversal of hyperglycemia was observed but the actual survivability of WJMSCs or WJMSC derived islet clusters were not assessed [91]. Since an inflammatory and an autoimmune component in the STZ model has not been reported clearly further research should focus on role of WJMSC and WJMSC islet derivatives in a physiological auto-immune diabetes scenario. The inflammatory component of physiological diabetes can be mimicked by changing the STZ dosage [93]. Undifferentiated BMMSCs when injected in STZ diabetic model rescued the glucose homeostasis [reviewed in 2]. However, the experiments were performed in immune compromised mice for BMMSCs in contrast to WJMSCs. Thus, the survivability and efficacy of WJMSCs and WJMSC generated islets need to be further tested out in other autoimmune disease models.

In contrast to the diabetic model, hUCMSCs transplanted in immuno-competent hemi-Parkison rats survived and caused disease recovery where as neurospheres generated from UCMCS ameliorated disease but did not survive post transplantations $[12,41]$. In both the studies recovery post injury was due to secretion of trophic factors and attenuation of microglial and astrocyte activation. So, the usage and transplant acceptance of UCMSCs or differentiated progenitors is also dependent on the disease context.

In addition, the immune-suppressive function of BMMSCs might be attenuated at higher passages. The immune modulatory potential of WJMSCs has not been tested beyond passage 10 to date. It is possible that continuous culturing results in expansion of subsets with distinct immune properties.

\section{PERSPECTIVES FOR THE FUTURE}

To summarize, WJMSCs have lucrative characteristics which make them promising candidates for regenerative cell therapy across MHC barriers. However, detailed studies need to be conducted to address the following issues before clinical extrapolation in humans.

a) A more global definition of WJMSCs is warranted. Contradictions in the literature could be due to different isolation procedures and enrichment of different populations at different passages. This can be established if clonal cultures are generated, individual clones are carefully characterized and a universal definition is coined based on these results.

b) Human WJMSC bio-distribution and homing post transplantation.

c) Clinical context in which WJMSCs are transplanted; MSCs can sustain or inhibit T cell responses depending on the tissue micro-environment and cellular/ cytokine milieu.

d) Xenogeneic systems might not be the optimal models to study immunogenicity of WJMSC. Most crucial

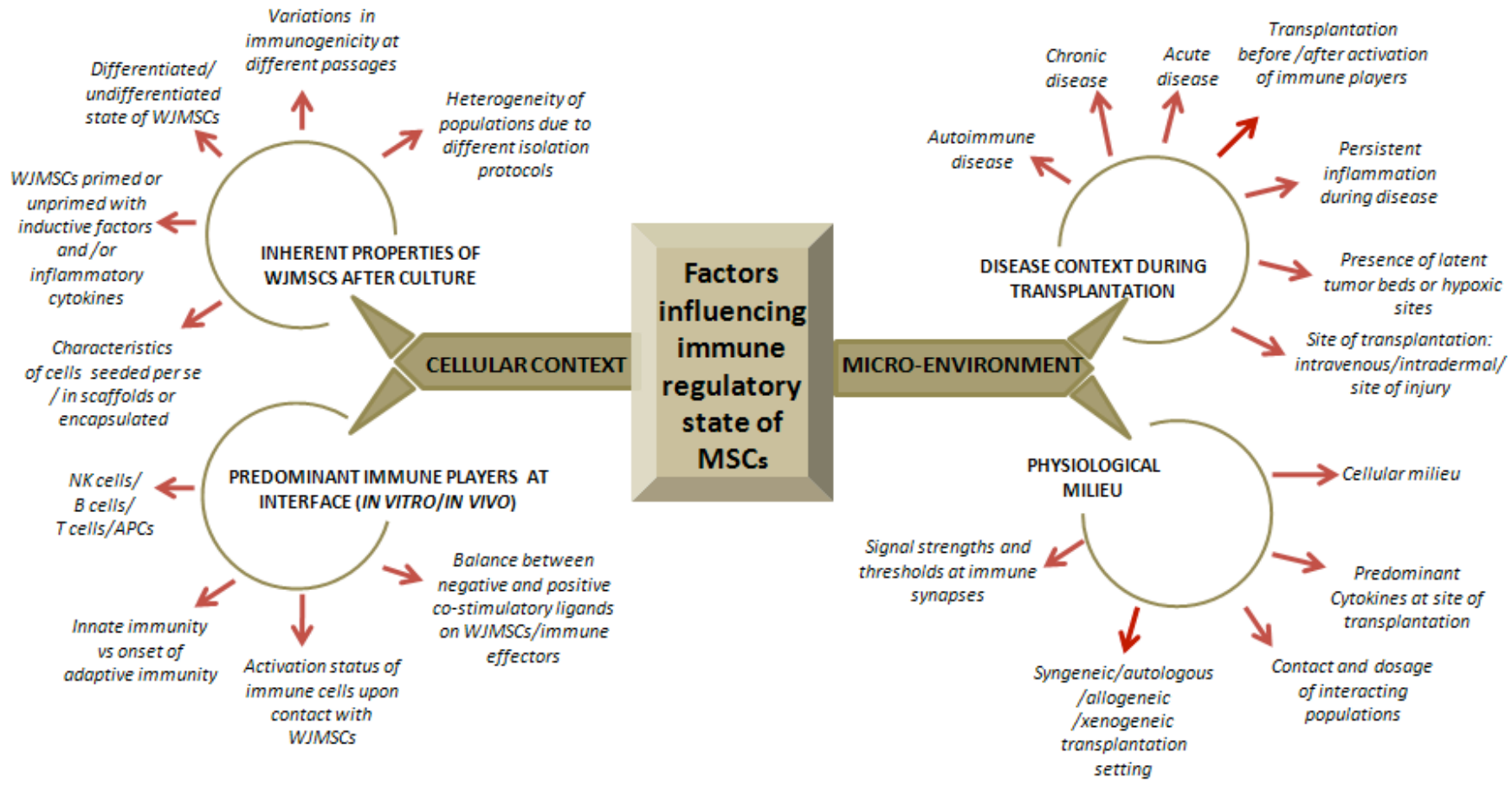

Fig. (2). Immune regulatory properties of WJMSCs are fined tuned by cellular and micro-environmental influences. 
cytokines like IFN $\gamma$ function in a species-specific manner and might prove inefficient at mouse-human interfaces resulting in transplant acceptance. It remains to be seen if results in preclinical models remain conserved when extrapolated to humans in the clinic.

e) During development of clinical protocols the immunogenicity of WJMSCs need to be assessed in context of site of injection, whether repeated injections are given at the same site or whether the disease results in a highly inflammatory environment.

f) The dosage and need of combining immunosuppressive regimens during WJMSC transplantation has to be evaluated specifically in situations where repeated injections are obligatory to achieve clinical benefit.

g) The efficacy of WJMSCs need to evaluated in different disease contexts; an autoimmune, chronic versus acute inflammation scenarios before putting it to translational use.

h) WJMSCs being more immune suppressive than their bone marrow counterparts can attenuate antitumor responses which generally eliminate benign tumors. Although occurrences of tumors have not been reported upon WJMSC transplantation, a prior existence of a latent tumor or a tumor history can pose problems.

A better understanding of the intrinsic characteristics of WJMSCs would help us decisively take a more confident step towards translational use of these cells as off the shelf therapeutics.

\section{ACKNOWLEDGEMENTS}

A special thanks to Professor Pawan Kumar Gupta and Anoop Babu Vasandan for critically evaluating the review and lending us technical support for the figures and the text.

\section{REFERENCES}

[1] Friedenstein AJ, Chailakhyan RK, Latsinik NV, Panasyuk AF, Keiliss-Borok IV. Stromal cells responsible for transferring the microenvironment of the hemopoietic tissues. Cloning in vitro and retransplantation in vivo. Transplantation 1974; 17(4):331-40.

[2] Abdi R, Fiorina P, Adra CN, Atkinson M, Sayegh MH. Immunomodulation by mesenchymal stem cells: a potential therapeutic strategy for type 1 diabetes. Diabetes 2008; 57(7): 1759-67.

[3] da Silva Meirelles L, Chagastelles PC, Nardi NB. Mesenchymal stem cells reside in virtually all post-natal organs and tissues. J Cell Sci 2006; 119(Pt 11): 2204-13.

[4] Hoogduijn MJ, Crop MJ, Peeters AM, et al. Human heart, spleen, and perirenal fat-derived mesenchymal stem cells have immunomodulatory capacities. Stem Cells Dev 2007; 16(4): 597604.

[5] Ghannam S, Bouffi C, Djouad F, Jorgensen C, Noël D. Immunosuppression by mesenchymal stem cells: mechanisms and clinical applications. Stem Cell Res Ther 2010; 1: 2.

[6] Götherström C, Ringdén O, Westgren M, Tammik C, Le Blanc K. Immunomodulatory effects of human fetal liver-derived mesenchymal stem cells. Bone Marrow Transplant 2003; 3: 26572 .

[7] Weiss ML, Anderson C, Medicetty S, et al. Immune properties of human umbilical cord Wharton's jelly-derived cells. Stem Cells 2008; 26(11): 2865-74.
[8] Rao MS, Mattson MP. Stem cells and aging: expanding the possibilities. Mech Ageing Dev 2001; 122(7): 713-34.

[9] Stenderup K, Justesen J, Clausen C, Kassem M. Aging is associated with decreased maximal life span and accelerated senescence of bone marrow stromal cells. Bone 2003; 33(6): 91926.

[10] Huang K, Zhou DH, Huang SL, Liang SH. Age-related biological characteristics of human bone marrow mesenchymal stem cells from different age donors. Zhongguo Shi Yan Xue Ye Xue Za Zhi 2005; 13(6): 1049-53.

[11] Stolzing A, Jones E, McGonagle D, Scutt A. Age-related changes in human bone marrow-derived mesenchymal stem cells: consequences for cell therapies. Mech Ageing Dev 2008; 129(3): 163-73.

[12] Weiss ML, Medicetty S, Bledsoe AR, et al. Human umbilical cord matrix stem cells: preliminary characterization and effect of transplantation in a rodent model of Parkinson's disease. Stem Cells 2006; 24(3): 781-92.

[13] Lu LL, Liu YJ, Yang SG, et al. Isolation and characterization of human umbilical cord mesenchymal stem cells with hematopoiesissupportive function and other potentials. Haematologica 2006; 91(8): 1017-26.

[14] Karahuseyinoglu S, Cinar O, Kilic E, et al. Biology of stem cells in human umbilical cord stroma: in situ and in vitro surveys. Stem Cells 2007; 25(2): 319-31.

[15] Can A, Karahuseyinoglu S. Concise review: human umbilical cord stroma with regard to the source of fetus-derived stem cells. Stem Cells 2007; 25(11): 2886-95.

[16] Troyer DL, Weiss ML. Wharton's jelly-derived cells are a primitive stromal cell population. Stem Cells 2008; 26(3): 591-9.

[17] Sarugaser R, Lickorish D, Baksh D, Hosseini MM, Davies JE. Human umbilical cord perivascular (HUCPV) cells: a source of mesenchymal progenitors. Stem Cells 2005; 23(2): 220-9.

[18] Lund RD, Wang S, Lu B, et al. Cells isolated from umbilical cord tissue rescue photoreceptors and visual functions in a rodent model of retinal disease. Stem Cells 2007; 3: 602-11.

[19] Nekanti U, Rao VB, Bahirvani AG, Jan M, Totey S, Ta M. Longterm expansion and pluripotent marker array analysis of Wharton's jelly-derived mesenchymal stem cells. Stem Cells Dev 2010; 19(1): 117-30.

[20] Prasanna SJ, Gopalakrishnan D, Shankar SR, Vasandan AB. Proinflammatory cytokines, IFNgamma and TNFalpha, influence immune properties of human bone marrow and Wharton jelly mesenchymal stem cells differentially. PLoS One 2010; 5(2): e9016.

[21] Pappa KI, Anagnou NP. Novel sources of fetal stem cells: where do they fit on the developmental continuum? Regen Med 2009; 4(3): 423-33.

[22] De Miguel MP, Arnalich Montiel F, Lopez Iglesias P, Blazquez Martinez A, Nistal M. Epiblast-derived stem cells in embryonic and adult tissues. Int J Dev Biol 2009; 53(8-10): 1529-40.

[23] Carlin R, Davis D, Weiss M, Schultz B, Troyer D. Expression of early transcription factors Oct-4, Sox-2 and Nanog by porcine umbilical cord (PUC) matrix cells. Reprod Biol Endocrinol 2006; 4: 8 .

[24] Zimmermann S, Voss M, Kaiser S, Kapp U, Waller CF, Martens UM. Lack of telomerase activity in human mesenchymal stem cells. Leukemia 2003; 17(6): 1146-9.

[25] La Rocca G, Anzalone R, Corrao S, et al. Isolation and characterization of Oct-4+/HLA-G+ mesenchymal stem cells from human umbilical cord matrix: differentiation potential and detection of new markers. Histochem Cell Biol 2009; 131(2): 26782.

[26] Fong CY, Chak LL, Biswas A, et al. Human Wharton's jelly stem cells have unique transcriptome profiles compared to human embryonic stem cells and other mesenchymal stem cells. Stem Cell Rev 2011; 7(1): 1-16.

[27] Anzalone R, Lo Iacono M, Corrao S, et al. New emerging potentials for human Wharton's jelly mesenchymal stem cells: immunological features and hepatocyte-like differentiative capacity. Stem Cells Dev 2010; 19(4): 423-38.

[28] Hsieh JY, Fu YS, Chang SJ, Tsuang YH, Wang HW. Functional module analysis reveals differential osteogenic and stemness potentials in human mesenchymal stem cells from bone marrow and Wharton's jelly of umbilical cord. Stem Cells Dev 2010; 19(12): 1895-910. 
[29] Alaminos M, Pérez-Köhler B, Garzón I, et al. Transdifferentiation potentiality of human Wharton's jelly stem cells towards vascular endothelial cells. J Cell Physiol 2010; 223(3): 640-7.

[30] Bongso A, Fong CY, Gauthaman K. Taking stem cells to the clinic: Major challenges. J Cell Biochem 2008; 105(6): 1352-60.

[31] Magin AS, Körfer NR, Partenheimer H, Lange C, Zander A, Noll T. Primary cells as feeder cells for coculture expansion of human hematopoietic stem cells from umbilical cord blood--a comparative study. Stem Cells Dev 2009; 18(1): 173-86.

[32] Kubota H, Avarbock MR, Brinster RL. Growth factors essential for self-renewal and expansion of mouse spermatogonial stem cells. Proc Natl Acad Sci USA 2004; 101(47): 16489-94.

[33] Sadler TW. Second week of development: Bilaminar germ disc. In: Langmann's Medical Embryology. Philadelphia: Williams \& Wilkins 2004; 51-64.

[34] Chao KC, Chao KF, Chen CF, Liu SH. A novel human stem cell coculture system that maintains the survival and function of culture islet-like cell clusters. Cell Transplant 2008; 17(6): 657-64.

[35] Dominici M, Le Blanc K, Mueller I, et al. Minimal criteria for defining multipotent mesenchymal stromal cells. The international society for cellular therapy position statement. Cytotherapy 2006; 8(4): 315-7.

[36] Baksh D, Yao R, Tuan RS. Comparison of proliferative and multilineage differentiation potential of human mesenchymal stem cells derived from umbilical cord and bone marrow. Stem Cells 2007; 6: 1384-92.

[37] Zhang ZY, Teoh SH, Chong MS, et al. Superior osteogenic capacity for bone tissue engineering of fetal compared with perinatal and adult mesenchymal stem cells. Stem Cells 2009; 1 : 126-37.

[38] Wang L, Ott L, Seshareddy K, Weiss ML, Detamore MS. Musculoskeletal tissue engineering with human umbilical cord mesenchymal stromal cells. Regen Med 2011; 1: 95-109.

[39] Karahuseyinoglu S, Kocaefe C, Balci D, Erdemli E, Can A. Functional structure of adipocytes differentiated from human umbilical cord stroma-derived stem cells. Stem Cells 2008; 26(3): 682-91.

[40] Hermann A, Gastl R, Liebau S, et al. Efficient generation of neural stem cell-like cells from adult human bone marrow stromal cells. J Cell Sci 2004; $117(\mathrm{Pt} 19)$ : 4411-22.

[41] Zhang L, Zhang HT, Hong SQ, Ma X, Jiang XD, Xu RX. Cografted Wharton's jelly cells-derived neurospheres and BDNF promote functional recovery after rat spinal cord transection. Neurochem Res 2009; 34(11): 2030-9.

[42] Mitchell KE, Weiss ML, Mitchell BM, et al. Matrix cells from Wharton's jelly form neurons and glia. Stem Cells 2003; 21(1): 5060.

[43] Fu YS, Shih YT, Cheng YC, Min MY. Transformation of human umbilical mesenchymal cells into neurons in vitro. J Biomed Sci 2004; 11(5): 652-60.

[44] Ma L, Feng XY, Cui BL, et al. Human umbilical cord Wharton's Jelly-derived mesenchymal stem cells differentiation into nervelike cells. Chin Med J (Engl) 2005; 118(23): 1987-93.

[45] Jomura S, Uy M, Mitchell K, Dallasen R, Bode CJ, Xu Y. Potential treatment of cerebral global ischemia with Oct-4+ umbilical cord matrix cells. Stem Cells 2007; 1: 98-106.

[46] Yang CC, Shih YH, Ko MH, Hsu SY, Cheng H, Fu YS. Transplantation of human umbilical mesenchymal stem cells from Wharton's jelly after complete transection of the rat spinal cord. PLoS One 2008; 3(10): e3336.

[47] Fu YS, Cheng YC, Lin MY, et al. Conversion of human umbilical cord mesenchymal stem cells in Wharton's jelly to dopaminergic neurons in vitro: potential therapeutic application for Parkinsonism. Stem Cells 2006; 1: 115-24

[48] Chao KC, Chao KF, Fu YS, Liu SH. Islet-like clusters derived from mesenchymal stem cells in Wharton's Jelly of the human umbilical cord for transplantation to control type 1 diabetes. PLoS One 2008; 1: e1451

[49] Zhang YN, Lie PC, Wei X. Differentiation of mesenchymal stromal cells derived from umbilical cord Wharton's jelly into hepatocyte-like cells. Cytotherapy 2009; 11(5): 548-58.

[50] Wu LF, Wang NN, Liu YS, Wei X. Differentiation of Wharton's jelly primitive stromal cells into insulin-producing cells in comparison with bone marrow mesenchymal stem cells. 2009; 15(10): 2865-73.
[51] Anzalone $\mathrm{R}$, Lo Iacono $\mathrm{M}$, Loria $\mathrm{T}$, et al. Wharton's Jelly mesenchymal stem cells as candidates for beta cells regeneration: extending the differentiative and immunomodulatory benefits of adult mesenchymal stem cells for the treatment of type 1 diabetes. Anzalone Stem Cell Rev 2011; 7(2): 342-63.

[52] Tsai PC, Fu TW, Chen YM, et al. The therapeutic potential of human umbilical mesenchymal stem cells from Wharton's jelly in the treatment of rat liver fibrosis. Liver Transpl 2009; 15(5): 48495.

[53] Klyushnenkova E, Mosca JD, Zernetkina V, et al. T cell responses to allogeneic human mesenchymal stem cells: immunogenicity, tolerance, and suppression. J Biomed Sci 2005; 12: 47-57.

[54] Le Blanc K, Tammik L, Sundberg B, Haynesworth SE, Ringdén O. Mesenchymal stem cells inhibit and stimulate mixed lymphocyte cultures and mitogenic responses independently of the major histocompatibility complex. Scand J Immunol 2003; 1: 11-20.

[55] Deuse T, Stubbendorff M, Tang-Quan K, et al. Immunogenicity and immunomodulatory properties of umbilical cord lining mesenchymal stem cells. Cell Transplant 2011; 20(5): 655-67.

[56] Tipnis S, Viswanathan C, Majumdar AS. Immunosuppressive properties of human umbilical cord-derived mesenchymal stem cells: role of B7-H1 and IDO. Immunol Cell Biol 2010; 88(8): 795806.

[57] Potian JA, Aviv H, Ponzio NM, Harrison JS, Rameshwar P. Vetolike activity of mesenchymal stem cells: functional discrimination between cellular responses to alloantigens and recall antigens. J Immunol 2003; 7: 3426-34.

[58] Eliopoulos N, Stagg J, Lejeune L, Pommey S, Galipeau J. Allogeneic marrow stromal cells are immune rejected by $\mathrm{MHC}$ class I- and class II-mismatched recipient mice. Blood 2005; 106(13): 4057-65.

[59] Djouad F, Plence P, Bony C, et al. Immunosuppressive effect of mesenchymal stem cells favors tumor growth in allogeneic animals. Blood 2003; 102(10): 3837-44.

[60] Liechty KW, MacKenzie TC, Shaaban AF, et al. Human mesenchymal stem cells engraft and demonstrate site-specific differentiation after in utero transplantation in sheep. Nat Med 2000; 6(11): 1282-6.

[61] Grinnemo KH, Kumagai-Braesch $\mathrm{M}$, Månsson-Broberg $\mathrm{A}$, et al. Human embryonic stem cells are immunogenic in allogeneic and xenogeneic settings. Reprod Biomed Online 2006; 13(5): 712-24.

[62] Swijnenburg RJ, Swijnenburg RJ, Tanaka M, et al. Embryonic stem cell immunogenicity increases upon differentiation after transplantation into ischemic myocardium. Circulation 2005; 112(9 Suppl): I166-72.

[63] Medicetty S, Bledsoe AR, Fahrenholtz CB, Troyer D, Weiss ML. Transplantation of pig stem cells into rat brain: proliferation during the first 8 weeks. Exp Neurol 2004; 1: 32-41.

[64] Di Nicola M, Carlo-Stella C, Magni M, et al. Human bone marrow stromal cells suppress T-lymphocyte proliferation induced by cellular or nonspecific mitogenic stimuli. Blood 2002; 10: 3838-43.

[65] Aggarwal S, Pittenger MF. Human mesenchymal stem cells modulate allogeneic immune cell responses. Blood 2005; 4: 181522.

[66] Najar M, Rouas R, Raicevic G, et al. Mesenchymal stromal cells promote or suppress the proliferation of $\mathrm{T}$ lymphocytes from cord blood and peripheral blood: the importance of low cell ratio and role of interleukin-6. Cytotherapy 2009; 11(5): 570-83.

[67] Puymirat E, Geha R, Tomescot A, et al. Can mesenchymal stem cells induce tolerance to cotransplanted human embryonic stem cells? Mol Ther 2009; 17(1): 176-82.

[68] Corcione A, Benvenuto F, Ferretti E, et al. Human mesenchymal stem cells modulate B-cell functions. Blood 2006; 107(1): 367-72.

[69] Nauta AJ, Westerhuis G, Kruisselbrink AB, Lurvink EG, Willemze R, Fibbe WE. Donor-derived mesenchymal stem cells are immunogenic in an allogeneic host and stimulate donor graft rejection in a nonmyeloablative setting. Blood 2006; 108(6): 211420 .

[70] Nauta AJ, Fibbe WE. Immunomodulatory properties of mesenchymal stromal cells. Blood 2007; 10: 3499-506.

[71] Meisel R, Zibert A, Laryea M, Göbel U, Däubener W, Dilloo D. Human bone marrow stromal cells inhibit allogeneic T-cell responses by indoleamine 2,3-dioxygenase-mediated tryptophan degradation. Blood 2004; 103(12): 4619-21.

[72] Tyndall A, Walker UA, Cope A, et al. Immunomodulatory properties of mesenchymal stem cells: a review based on an 
interdisciplinary meeting held at the Kennedy Institute of Rheumatology Division, London, UK, 31 October 2005.Tyndall arthritis research therapy. Arthritis Res Ther 2007; 9(1): 301.

[73] Ren G, Zhang L, Zhao X, et al. Mesenchymal stem cell-mediated immunosuppression occurs via concerted action of chemokines and nitric oxide. Cell Stem Cell 2008; 2: 141-50.

[74] Li H, Guo Z, Jiang X, Zhu H, Li X, Mao N. Mesenchymal stem cells alter migratory property of $\mathrm{T}$ and dendritic cells to delay the development of murine lethal acute graft-versus-host disease. Stem Cells 2008; 26(10): 2531-41.

[75] Carosella ED, Moreau P, Le Maoult J, Le Discorde M, Dausset J, Rouas-Freiss N. HLA-G molecules: from maternal-fetal tolerance to tissue acceptance. Adv Immunol 2003; 81: 199-252.

[76] Vogiagis D, Salamonsen LA. The role of leukaemia inhibitory factor in the establishment of pregnancy. J Endocrinol 1999; 160(2): 181-90.

[77] Najar M, Raicevic G, Boufker HI, et al. Adipose-tissue-derived and Wharton's jelly-derived mesenchymal stromal cells suppress lymphocyte responses by secreting leukemia inhibitory factor. Tissue Eng Part A 2010; 16(11): 3537-46.

[78] Selmani Z, Naji A, Zidi I, et al. Human leukocyte antigen-G5 secretion by human mesenchymal stem cells is required to suppress $\mathrm{T}$ lymphocyte and natural killer function and to induce CD4+CD25 high FOXP3+ regulatory T cells. Stem Cells 2008; 26(1): 212-22.

[79] Yoo KH, Jang IK, Lee MW, et al. Comparison of immunomodulatory properties of mesenchymal stem cells derived from adult human tissues. Cell Immunol 2009; 259(2): 150-6.

[80] Fang L, Lange C, Engel M, Zander AR, Fehse B. Sensitive balance of suppressing and activating effects of mesenchymal stem cells on T-cell proliferation. Transplantation 2006; 82(10): 1370-3.

[81] Magatti M, De Munari S, Vertua E, Gibelli L, Wengler GS, Parolini O. Human amnion mesenchyme harbors cells with allogeneic T-cell suppression and stimulation capabilities. Stem Cells 2008; 26(1): 182-92.

[82] Krampera M, Cosmi L, Angeli R, et al. Role for interferon-gamma in the immunomodulatory activity of human bone marrow mesenchymal stem cells. Stem Cells 2006; 2: 386-98.

[83] Polchert D, Sobinsky J, Douglas G, et al. IFN-gamma activation of mesenchymal stem cells for treatment and prevention of graft versus host disease. Eur J Immunol 2008; 38(6): 1745-55.
[84] Cho PS, Messina DJ, Hirsh EL, et al. Immunogenicity of umbilical cord tissue derived cells. Blood 2008; 111(1): 430-8.

[85] Lutton BV, Cho PS, Hirsh EL, et al. Approaches to avoid immune responses induced by repeated subcutaneous injections of allogeneic umbilical cord tissue-derived cells. Transplantation 2010; 90(5): 494-501.

[86] English K, Barry FP, Field-Corbett CP, Mahon BP. IFN-gamma and TNF-alpha differentially regulate immunomodulation by murine mesenchymal stem cells. Immunol Lett 2007; 110(2): 91100.

[87] Rasmusson I, Ringdén O, Sundberg B, Le Blanc K. Mesenchymal stem cells inhibit the formation of cytotoxic T lymphocytes, but not activated cytotoxic $\mathrm{T}$ lymphocytes or natural killer cells. Transplantation 2003; 76(8): 1208-13.

[88] Spaggiari GM, Capobianco A, Becchetti S, Mingari MC, Moretta L. Mesenchymal stem cell-natural killer cell interactions: evidence that activated NK cells are capable of killing MSCs, whereas MSCs can inhibit IL-2-induced NK-cell proliferation. Blood 2006; 107(4): 1484-90.

[89] Götherström C, Lundqvist A, Duprez IR, Childs R, Berg L, le Blanc K. Fetal and adult multipotent mesenchymal stromal cells are killed by different pathways. Cytotherapy 2011; 13(3): 269-78.

[90] Boissel L, Tuncer HH, Betancur M, Wolfberg A, Klingemann H. Umbilical cord mesenchymal stem cells increase expansion of cord blood natural killer cells. Biol Blood Marrow Transplant 2008; 14(9): 1031-8.

[91] Kadam SS, Bhonde RR. Islet neogenesis from the constitutively nestin expressing human umbilical cord matrix derived mesenchymal stem cells. Islets 2010; 2(2): 112-20.

[92] Wang HS, Shyu JF, Shen WS, et al. Transplantation of insulin producing cells derived from umbilical cord stromal mesenchymal stem cells to treat NOD mice. Cell Transplant 2010; [Epub ahead of print].

[93] Herold KC, Baumann E, Vezys V, Buckingham F. Expression and immune response to islet antigens following treatment with low doses of streptozotocin in H-2d mice. J Autoimmun 1997; 10(1): $17-25$.

(C) Prasanna and Jahnavi; Licensee Bentham Open.

This is an open access article licensed under the terms of the Creative Commons Attribution Non-Commercial License (http://creativecommons.org/ licenses/by-nc/3.0/), which permits unrestricted, non-commercial use, distribution and reproduction in any medium, provided the work is properly cited. 